\title{
Editorial
}

\section{Uncertainty-Based Design Optimization in Engineering: Model, Algorithm, and Application}

\author{
Xiaojun Wang, ${ }^{1}$ Zhiping Qiu, ${ }^{1}$ and Dirk Söffker ${ }^{2}$ \\ ${ }^{1}$ Institute of Solid Mechanics, Beihang University, Beijing 100191, China \\ ${ }^{2}$ Department of Mechanical Engineering, University of Duisburg-Essen, 47048 Duisburg, Germany \\ Correspondence should be addressed to Zhiping Qiu; zpqiu@buaa.edu.cn
}

Received 25 September 2013; Accepted 25 September 2013

Copyright (C) 2013 Xiaojun Wang et al. This is an open access article distributed under the Creative Commons Attribution License, which permits unrestricted use, distribution, and reproduction in any medium, provided the original work is properly cited.

Uncertainty widely exists in inherent characteristics or external environment of practical engineering problems. The comprehensive influence of uncertainty on products has already been one of the most significant evaluation criterions to better guide the subsequent R\&D design. Thus, the uncertaintybased design optimization (UBDO) will become an inexorable trend and main attention in the fields of academic study and engineering applications.

Three parts should be emphasized particularly in UBDOE. The first is the construction of uncertain analytical model, that is, to estimate/quantify uncertainty and further make it embody in the objective function or constraint condition of optimal process. Next in importance is the development of algorithm. Efficiency, convergence and stability are all needed to take account of. The third is the combination of the theoretical approaches and practical problems, namely, to formulate mathematical system reasonably from true environment. This issue complies 17 existing papers, most of which are commendably performed with reference to one of the above 3 aspects.

Four of the papers deal with the first aspect in great depth. Ş. Şeker et al. proposed a weighted multiobjective optimization method for mixed-model assembly line problem based on a mixed integer programming technique. A. Saavedra et al. established a generalized linear spatial model (GLSM) to explore relationship between exploitability and different explanatory variables that characterize slate quality. A time-dependent reliability-based design optimization (RBDO) method by Y. Wang et al. was developed to consider both the time-dependent kinematic reliability and the timedependent structural reliability as constrains. In this study, a nonintrusive time-dependent surrogate model derived from polynomial chaos with the moving least squares (PCMLS) was proposed to achieve uncertainty quantification. Furthermore, the paper authored by Y. Lei et al. presented a probabilistic approach for the identification of structural damage and unknown external excitations using only limited measurements of structural acceleration responses contaminated by intensive measurement noises.

The research on the second algorithm is addressed on two papers, one of which by P. Tontragunrat and S. Bureerat investigated a metaheuristic method by virtue of population-based incremental learning (PBIL) to solve the anti-optimization problem subjected to the truss structure. Another one is contributed by Y. Li and C.-L. Zheng who launched an investigation in the complex network synchronization via chaos control nodes. They adopted different order chaotic systems as the nodes for complex network with the help of symbolic computation, and the structure of coupling functions among the connected nodes was obtained based on Lyapunov stability theory.

In the aspect of engineering application, a fuzzy multiobjective design model used to minimize the length of traffic queue and pedestrian delays is proposed by N. S. Pour et al., who also introduced the genetic algorithm and a new defuzzification method. Treatments in other fields, including by principal component pursuit (PCP) as well as estimation of stock return are, respectively, studied by Q. You and G. Cao. 
We sincerely hope that our efforts by compiling these papers can enrich our readers and inspire researchers with regard to the seemingly common but actually important issue of UBDOE.

Xiaojun Wang

Zhiping Qiu Dirk Söffker 


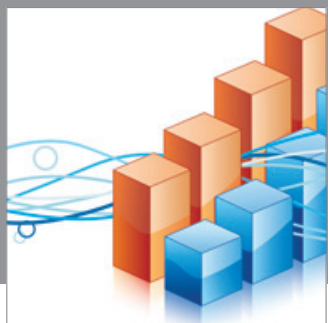

Advances in

Operations Research

mansans

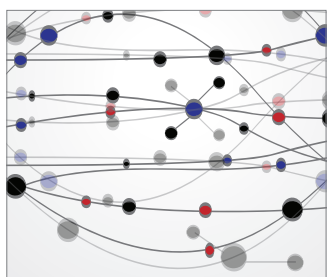

The Scientific World Journal
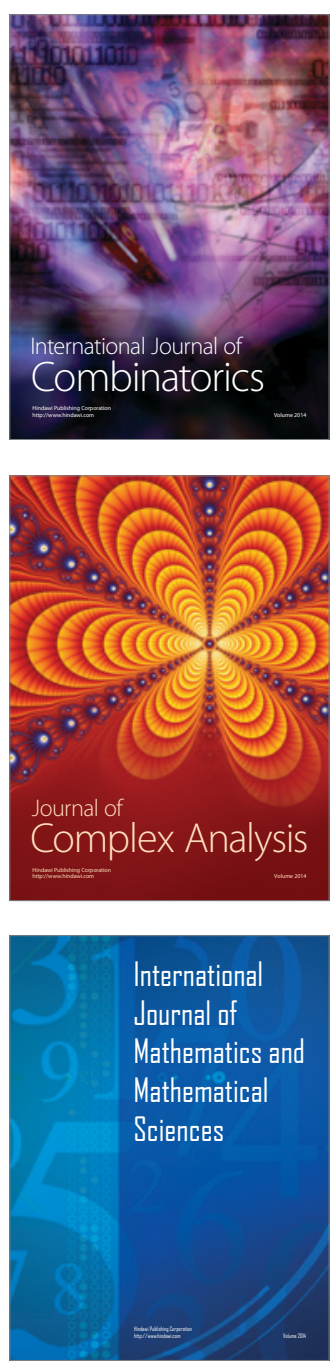
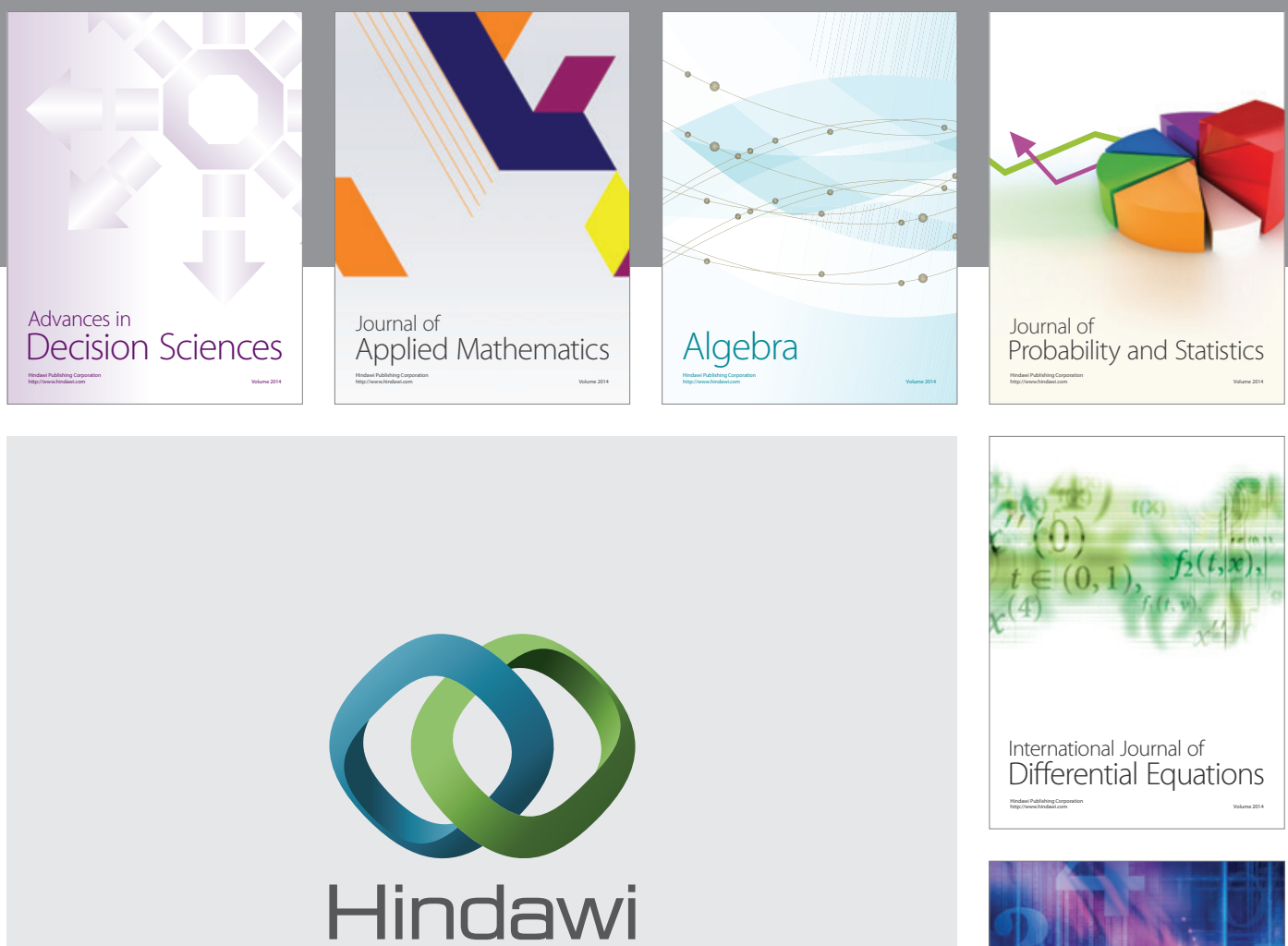

Submit your manuscripts at http://www.hindawi.com
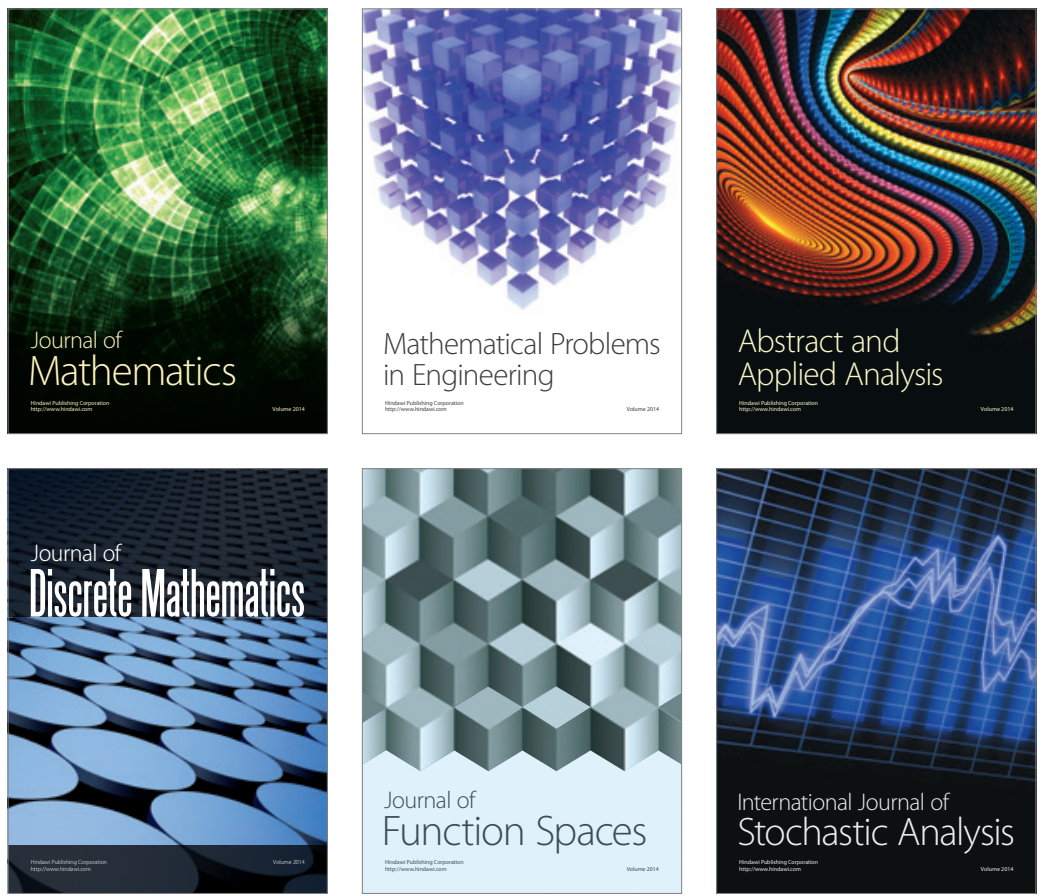

Journal of

Function Spaces

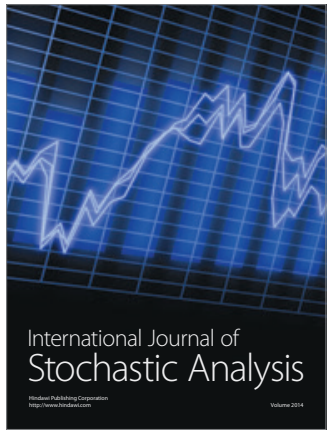

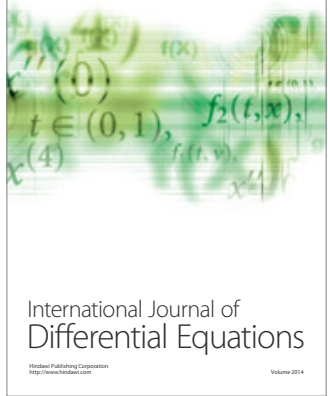
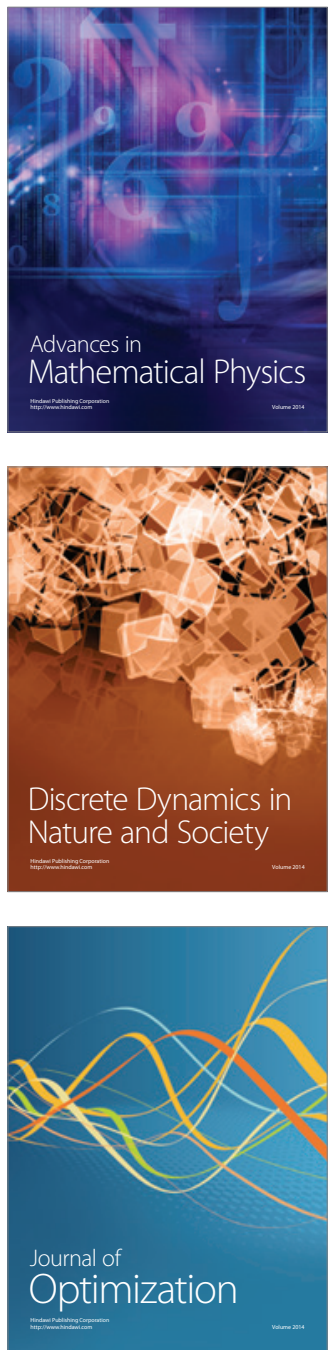